IFAS Extension

\title{
Sensory Acceptability of Puréed Foods ${ }^{1}$
}

Jamila R. Lepore and Wendy J. Dahl ${ }^{2}$

\section{Sensory Evaluation - What is it?}

Sensory evaluation is the process of using our senses (taste, smell, touch, sight) and applying them to determine the acceptability of foods. Simply, it is assessing the food to make sure that it looks, smells, and tastes delicious!

\section{Why evaluate the acceptability of puréed foods?}

It is important that we enjoy the food we eat. This makes for a better quality of life. This is no different for those people needing to be on puréed diets due to swallowing problems $(1,2)$. Taste and aroma are even more important with puréed foods because of the modification of texture and appearance.

All puréed foods should be evaluated before serving, new recipes in particular. Just like you wouldn't want to serve guests a recipe without trying it first, you don't want to serve a puréed food without knowing that it is appealing.

\section{Evaluating Puréed Foods}

The chef/cook, clients, or both can sample puréed foods. For best results, test new recipes by having those who are consuming the foods evaluate them so that the cook/chef can make changes as needed. An easy way to do this is to have a discussion group. Present a new purée, and then have the group rate it and provide feedback.

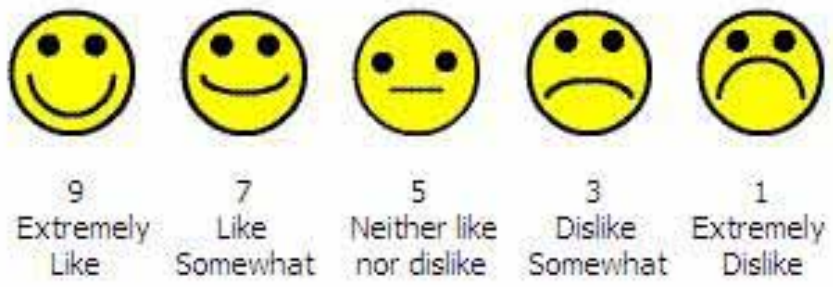

Figure 1. Rating scale with five distinct degrees of satisfaction illustrated by easily recognizable facial expressions ranging from classic smiley face (Extremely Like) to frowny face (Extremely Dislike)

A straightforward rating scale that can be used is a 9-point scale where $1=$ extremely dislike, $5=$ neither like nor dislike, and $9=$ extremely like. Using images makes it even simpler.

Questions to ask using this scale:

- How would you rate taste?

- How would you rate aroma?

- How would you rate appearance?

- How would you rate texture?

Rating scales allow you to compare between foods and between individuals.

Between Foods: For example, a recipe for puréed broccoli may get an average rating of 5 while one for carrots gets an

1. This document is FSHN12-13, one in a series of the Food Science and Human Nutrition Department, Florida Cooperative Extension Service, Institute of Food and Agricultural Sciences, University of Florida. Published November 2012. Visit the EDIS website at http://edis.ifas.ufl.edu/.

2. Jamila R. Lepore, RD, graduate student; Wendy J. Dahl, PhD, RD, assistant professor; Food Science and Human Nutrition Department, Institute of Food and Agricultural Sciences, University of Florida, Gainesville, FL 32611. 
average rating of 7 . This suggests that the broccoli recipe may need to be adjusted to improve its flavor. Alternatively, it is possible that carrots are simply well-liked and broccoli less so. You will need to consider what average ranking would be considered unacceptable.

Between Individuals: For example, a group of taste-testers ranks puréed peaches an average of 8 but one person ranks them as 1 . You may want to determine if he/she simply does not like peaches, or perhaps misunderstood the ranking scale. Despite the one low ranking, this is a recipe that should be used and a food that is offered because the majority of the group ranked it highly.

Attached at the end of this document is a sensory scale from which you can make copies to evaluate your purées.

\section{Tips for Improving Puréed Foods}

\section{Taste}

The taste of puréed foods needs to be more intense than for regular texture foods. This is because the act of chewing a food releases flavors in the mouth. Once a food is puréed, it does not require chewing. It can be simply swallowed without much time for the flavor of the food to be tasted. Increasing the flavor of puréed foods helps with flavor recognition.

There are several ways to achieve a stronger and improved flavor:

- Flavor enhancers are food ingredients that can be used to increase the natural flavor of a food item. They increase flavor intensity. These are special ingredients that can be purchased. Older adults have been shown to have decreased sensation of taste and smell $(3,4)$. Flavor enhancers have been shown to improve food intakes of elderly persons (5). Examples of flavor enhancers include (6):
- When adjusting the consistency of puréed foods, try using flavorful alternatives as your liquid instead of water. For instance, use flavored broth or gravy with meats, fruit juice for fruit purées, and milk for dairy products and desserts.

- Spices and herbs can also be added to puréed foods to increase acceptability. However, these alter the natural flavors of food and may make it harder to identify puréed foods.

There are also things to keep in mind that can negatively impact flavor (7). These include refrigeration and freezing, microwaving, and reheating of foods.

These methods of storage and preparation can produce off-flavors, especially with foods containing fats such as many meat, seafood, and dairy products (7). While these processes are often necessary in food production, there are a few things that can be done to maximize pleasant flavors while minimizing off-flavor.

- Whenever possible, prepare purées with the freshest ingredients possible and serve immediately. If you do need to prepare items a day or two in advance, it is better to freeze than to refrigerate.

- When refrigeration and storage are inevitable, limit the length of time food spends in storage. This is especially true for foods that have already been prepared, like purées. Keep storage time of house-made purées as short as possible. Commercially-prepared purées are designed to be frozen and may be stored longer.

- It may not be practical for you to create and serve purées immediately, so reheating is unavoidable. When reheating foods, it is best to prepare them using conventional methods (such as with the stovetop or oven) that will produce the best quality purée.

- Avoid using the microwave to ensure the best quality in your purées. Heating and reheating in a microwave can lead to off-flavors, especially with meats and seafood. Restrict the use of a microwave to products that are specifically designed for microwave heating, such as frozen vegetables or dishes that have instructions for microwave use.

It is also important not to combine puréed foods as the flavors may become unrecognizable. The inability to identify a food (e.g., is this chicken or pork?) has been 
associated with a negative meal time experience for those on puréed diets (8). For example, it is better to present purées as individual items or layers instead of mixing them into one "casserole" type item. For example, puréed pasta, puréed meatballs and tomato purée could be layered so that each flavor can be recognized. It is important to remember that when texture is modified, reliance on taste for food recognition is heightened.

\section{Aroma}

Aroma is one of the first senses we use during mealtimes. It is often the first thing we notice, even before the plate comes to the table. This is why it is so important to make sure the puréed foods smell good! Just like with taste, it is best to limit the number of foods combined in a puréed food so that the food aroma is recognizable. Think about it. If you smell ham but you eat a purée made of ham, potatoes, and green beans, it will probably taste unpleasant because it is not what was expected. This is because aroma is inherently linked to taste.

When a food is heated, the aroma is released. Aromas are best preserved when foods are heated by conventional means (stovetop, oven). Although microwaves are well suited for reheating puréed foods, aroma may be altered. Because microwaves heat unevenly, aroma is released at different times in different places on the food. As a result, some aromas are lost in the microwave, while others remain in the food. This alters the overall odor profile of the food when it is consumed (9).

It is also possible to alter the aroma of a food by the addition of ingredients that produce a potent aroma. A food that is puréed may have its aroma enhanced by the addition of herbs, spices and/or extracts (e.g. vanilla). Commercial aroma enhancers are available, the most common being monosodium glutamate (MSG) and 5 '-inosine monophosphate (5'IMP) (10).

\section{Texture}

Acceptable texture is one of the most important features of puréed foods! There are several things to keep in mind when preparing a puréed food to a proper consistency.

Make sure puréed foods are smooth and cohesive. A purée should not require chewing and should be "spoon-thick." It should not be too dry or too thin. Purées should not contain lumps.

Puréed foods should not be sticky. For example, mashed potatoes and other starchy puréed foods can often be

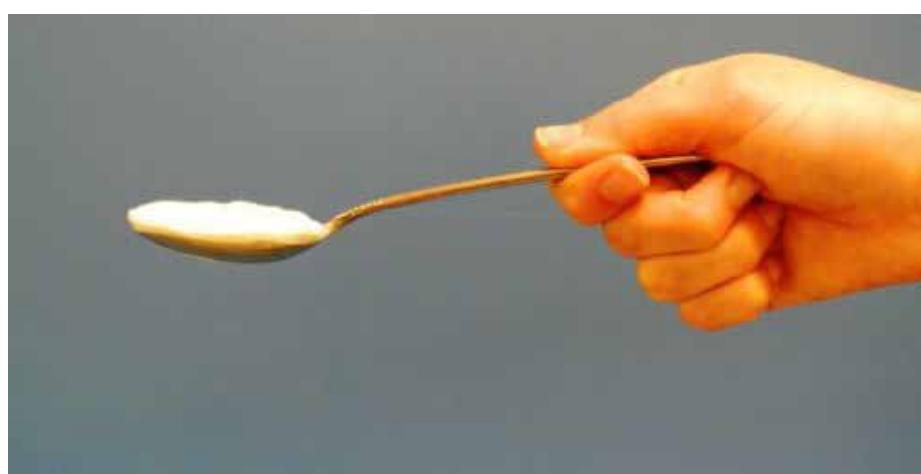

Figure 2. "Spoon thick"

Credits: UF/IFAS photo

thick and stick to the roof of the mouth. Adding gravy or sour cream can reduce stickiness and produce a smoother creamier texture. To decrease stickiness of starchy puréed food, use sauces, cream or other fats appropriate for the item being prepared.

Puréed foods should not be too thin. Thin purées are generally more difficult to swallow than spoon-thick purées. A purée that is too thin is often the result of adding too much liquid during preparation. To achieve the appropriate spoon-thick texture, add liquid a little bit at a time.

When preparing puréed foods, you may need to use a thickener. While there are many commercial starch thickeners available (e.g. Thick-It ${ }^{\oplus}$, Thickenup ${ }^{\circledR}$ ), using foods to thicken purées produces a product that is more nutritious and generally more appetizing. Grains work well for thickening purées. Some examples of food thickeners include:

\author{
Powdered crackers/bread crumbs \\ Baby cereal \\ Finely ground breakfast cereals \\ Puréed chickpeas or navy beans \\ Puréed bananas \\ Puréed starchy vegetables (potato, peas)
}

\section{Appearance}

Appearance is just as important as the other food characteristics. We often 'eat with our eyes' first. Color becomes more important with puréed food, as appearance is significantly altered.

It has been shown that older adults rely more on visual cues to identify food products (11). Recognition of food items is an important part of the mealtime experience for elderly on purée diets (8). Because a puréed food no longer has its original form, there are a few ways to make it more recognizable and appealing. 
Keep foods separate as much as possible. For instance, combining puréed carrots with puréed peas will produce an unattractive color. By keeping foods separate, food items retain their color and are more easily identifiable.

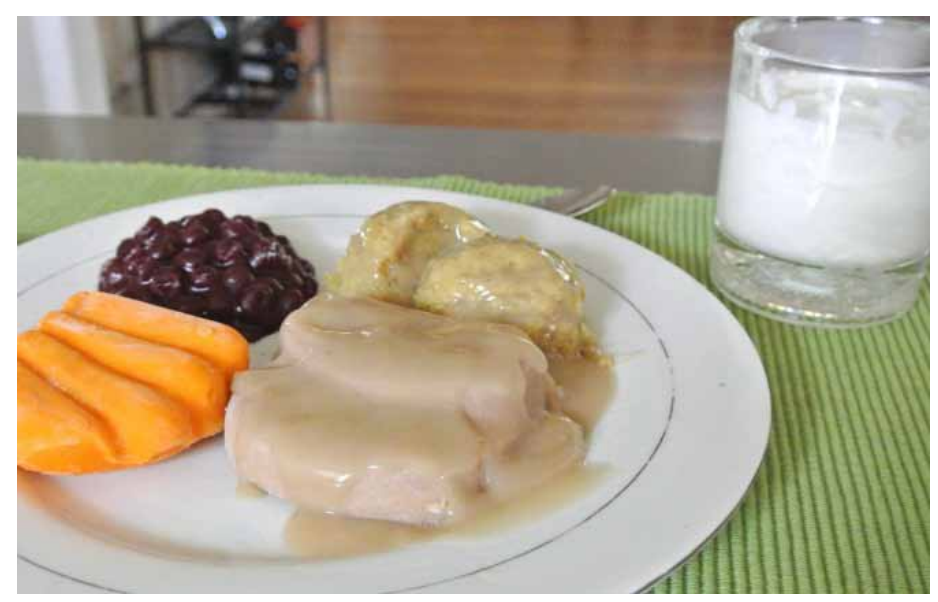

Figure 3. Meal of puréed food items, each molded separately to resemble the whole food item

Credits: UF/IFAS photo

Consider using food molds for purées. These molds are designed to make the purée resemble the original whole food item. The use of molds may or may not be preferred. Food molds can enhance appearance and improve intake for some individuals (12). For others, a natural unmolded purée is preferred. It is possible that the molded purée, when shaped like the original food, will lead to an expectation that the puréed food will have the same mouth feel as the original food. Make sure you ask if molded purées are preferred.

Adding puréed garnishes can enhance the appearance of purées as well. This is especially true if you are using scoops instead of food molds. Puréed garnishes include:

Sauces/Gravies
Condiments (ketchup, mustard, mayo, etc.)
Butter/Margarine
Sour Cream
Whipped Cream
Thick Syrups

\section{Summary \& Useful Links}

When trying new recipes or using existing recipes for purées, make sure it appeals to all of the senses. The purée should smell, taste, and look appetizing. It is also important that the texture be smooth and cohesive. The best way to evaluate whether your recipe entices with all of these qualities is to do sensory testing! This can be done by the chef, caregiver, and/or the person(s) with swallowing problems. Please see the attached form for your use when evaluating purées.

Listed here are useful publications for your reference:

- Puréed Foods for Swallowing Problems (1)

- Swallowing Problems and the Older Adult (2)

\section{References}

(1) Dahl WJ. Puréed Foods for Swallowing Problems. 2011 [cited; Available from: http://edis.ifas.ufl.edu/pdffiles/FS/ FS16800.pdf

(2) Dahl WJ. Swallowing Problems and the Older Adult. 2011 [cited; Available from: http://edis.ifas.ufl.edu/pdffiles/ FS/FS16400.pdf

(3) Schiffman SS, Warwick ZS. Use of flavor-amplified foods to improve nutritional-status in elderly persons. Annals of the New York Academy of Sciences. 1989;561:267-76.

(4) Schiffman SS, Warwick ZS. Effect of flavor enhancement of foods for the elderly on nutritional status, food intake, biochemical indexes, and anthropometric measures.

Physiology \& Behavior. 1993;53:395-402.

(5) Mathey M-F, Siebelink E, de Graaf C, Van Staveren WA. Flavor enhancement of foods improves dietary intake and nutritional status of elderly nursing home residents. Journal of Gerontology. 2001;56A:M200-5.

(6) Administration FaD. Food Additives: Alphabetical List. 2002.

(7) Flavor, Fragrance, and Odor Analysis. New York: Marcel Dekker, Inc.; 2002.

(8) Blaise M. Mealtime experiences of hospitalized older patients requiring a puree consistency diet: University of Montreal; 2009.

(9) Roberts DD, Pollien P. Analysis of Aroma Release during Microwave Heating. Journal of Agriculture \& Food Chemistry. 1997;45:4388-92.

(10) Astray G, García-Ríob L, Mejuto J, Pastrana L. Chemistry in Food: Flavours. Electronic Journal of Environmental, Agricultural, and Food Chemistry. 2007;6:1742-63.

(11) Philipsen DH, Clydesdale FM, Griffin RW, Stern P. Consumer age affects response to sensory characteristics of a cherry flavored beverage. Journal of Food Science. 1995;60:364-8.

(12) Cassens D, Johnson E, Keelan S. Enhancing taste, texture, appearance, and presentation of pureed food improved resident quality of life and weight status. Nutrition Reviews. 1996;54:S51-4. 
Food Evaluation Form

\begin{tabular}{|c|c|c|c|c|c|c|}
\hline Purée & Appearance & Aroma & Taste & Texture & Overall & Comments \\
\hline & & & & & & \\
\hline & & & & & & \\
\hline & & & & & & \\
\hline & & & & & & \\
\hline & & & & & & \\
\hline & & & & & & \\
\hline & & & & & & \\
\hline & Atromely it & (n) & $\begin{array}{l}\text { cale: } \\
\text { re Nor }\end{array}$ & Dislike & $/ 1=$ Extr & \\
\hline
\end{tabular}

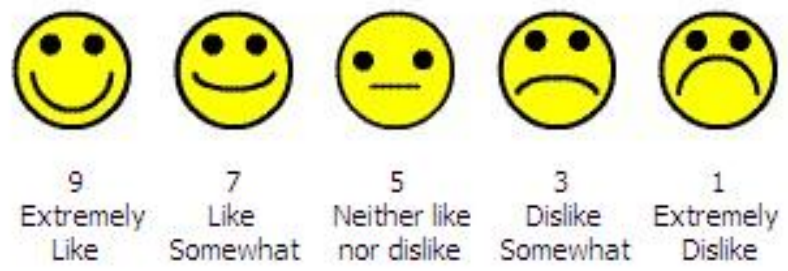

